\title{
Social Vulnerability as a Predictor of Physical Activity and Screen Time in European Children
}

Iguacel, Isabel; Fernández-Alvira, Juan M.; Bammann, Karin; Chadjigeorgiou, Charalambos; de Henauw, Stefaan; Heidinger-Felső, Regina; Lissner, Lauren; Michels, Nathalie; Page, Angie; Reisch, Lucia A.; Russo, Paola; Sprengeler, Ole; Veidebaum, Toomas; Börnhorst, Claudia; Moreno, Luis A.

Document Version

Accepted author manuscript

Published in:

International Journal of Public Health

DOI:

10.1007/s00038-017-1048-4

Publication date:

2018

License

Unspecified

Citation for published version (APA):

Iguacel, I., Fernández-Alvira, J. M., Bammann, K., Chadjigeorgiou, C., de Henauw, S., Heidinger-Felső, R., Lissner, L., Michels, N., Page, A., Reisch, L. A., Russo, P., Sprengeler, O., Veidebaum, T., Börnhorst, C., \& Moreno, L. A. (2018). Social Vulnerability as a Predictor of Physical Activity and Screen Time in European Children. International Journal of Public Health, 63(2), 283-295. https://doi.org/10.1007/s00038-017-1048-4

Link to publication in CBS Research Portal

\section{General rights}

Copyright and moral rights for the publications made accessible in the public portal are retained by the authors and/or other copyright owners and it is a condition of accessing publications that users recognise and abide by the legal requirements associated with these rights.

\section{Take down policy}

If you believe that this document breaches copyright please contact us (research.lib@cbs.dk) providing details, and we will remove access to the work immediately and investigate your claim. 


\section{Social Vulnerability as a Predictor of Physical Activity and Screen Time in European Children}

Isabel Iguacel, Juan M. Fernández-Alvira, Karin Bammann, Charalambos Chadjigeorgiou, Stefaan de Henauw, Regina Heidinger-Felső, Lauren Lissner, Nathalie Michels, Angie Page, Lucia A. Reisch, Paola Russo, Ole Sprengeler, Toomas Veidebaum, Claudia Börnhorst, and Luis A. Moreno

Journal article (Accepted version)

ClTE: Iguacel, I., Fernández-Alvira, J. M., Bammann, K., Chadjigeorgiou, C., de Henauw, S., Heidinger-Felső, R., ... Moreno, L. A. (2018). Social Vulnerability as a Predictor of Physical Activity and Screen Time in European Children. International Journal of Public Health, 632), 283-295. 001: 10.1007/s00038-017-1048-4

This is a post-peer-review, pre-copyedit version of an article published in International Journal of Public Health. The final authenticated version is available online at: https://doi.org/10.1007/s00038-017-1048-4

Uploaded to CBS Research Portal: January २०19 
1 Social vulnerability as a predictor of physical activity and screen time in European

2 children

3

4 Abstract

5

6 Objectives

7 To examine associations between social vulnerabilities and meeting physical activity

8 (PA) and screen time (ST) recommendations during a 2-year follow-up.

10 Methods

$11 \quad 13,891$ children aged 2.0-<9.9 from eight European countries were assessed at baseline 12 and 8,482 children at follow-up. Children's sports club membership, PA and ST were 13 collected via parental questionnaires. Moderate-to-vigorous physical activity (MVPA) 14 was objectively-assessed with accelerometers. Performing at least one hour of MVPA 15 daily and spending less than two hours of ST defined physically-active and non-sedentary 16 children respectively. Vulnerable groups were defined at baseline as children whose 17 parents had minimal social networks, from non-traditional families, with migrant origin 18 or with unemployed parents. Logistic mixed-effects analyses were performed adjusting 19 for classical socioeconomic indicators.

20

\section{Results}

22 Children whose parents had minimal social networks had a higher risk of non-compliance 23 with PA recommendations (subjectively-assessed) at baseline. Migrants and children with 24 unemployed parents had longer ST. All vulnerable groups were less likely to be sports 25 club members.

Conclusions

28 Migrants and children with unemployed parents are at risk for excessive ST and all vulnerable groups have lower odds of being sports club members.

31 Keywords:

32 Vulnerable groups

33 Physical activity

34 Accelerometry

35 Screen time 
Children

IDEFICS study

Abbreviations: PA, Physical Activity; MVPA, Moderate to Vigorous Physical Activity;

IDEFICS, Identification and prevention of Dietary- and lifestyle-induced Health EFfects in Children and infantS; ST, Screen Time; SES, Socio-Economic Status; T0, baseline; $\mathrm{T} 1$, follow-up after the intervention

\section{Introduction}

Regular physical activity (PA) during childhood is associated with improved musculoskeletal and cardiovascular health and lower adiposity (Janssen and Leblanc 2010; Strong et al. 2005). Insufficient PA and excessive screen time (ST) are independently associated with negative health outcomes (Ekelund et al. 2012). Therefore, increasing PA and decreasing sedentary time are public health priorities. Current guidelines for children aged 5 to 18 recommend at least one hour of moderate-to-vigorous physical activity (MVPA) per day (World Health Organization 2010) and to limit ST to no more than two hours a day (American Academy of Pediatrics 2001). Despite these benefits, many children do not meet the recommended level of PA or ST (Konstabel et al. 2014).

Socio-economic status (SES) is an important determinant of health in adults but results for children and adolescents are less consistent (Drenowatz et al. 2010). Some studies showed that youth from higher SES are more physically active than youth from lower SES (Hanson and Chen 2007) while one study in China reported that high SES was positively associated with insufficient PA (Wang et al. 2016). One reason for these differences may be that associations may vary by domain of PA. The association between SES and sport may be different to that for active transport, both of which contribute to overall PA. Regarding ST results also seem ambiguous (Pate et al. 2011). Similarly, studies vary according to the sedentary measure used. Some studies showed children from high SES groups spent more time on non-screen sedentary behaviours (such as sitting or lying down) and those from low SES spent more time in screen-based sedentary behaviours (e.g. watching TV). However, no significant differences between children from low and high SES backgrounds were found for total sedentary time (sum of nonscreen sedentary behaviours and ST) (Klitsie et al. 2013). Using subjective and objective 
methods, Foley et al. (Foley et al. 2011) showed that, children and adolescents in New Zealand from areas of lower deprivation (i.e., higher SES) accumulated more total sedentary time than those from higher deprivation. Inconsistent findings could be partially due to differential methods used to assess PA levels and sedentary time by e.g. subjective procedures such as questionnaires compared to objectives measures, such as accelerometers (Raudsepp and Viira 2008). Accelerometers are more accurate at assessing total time spent engaging in PA at different intensity levels and recording inactive time (Hagströmer et al. 2010). However, questionnaires are preferable to assess domains of PA (e.g. transport, sport, leisure) and sedentary-related behaviours (Atkin et al. 2012).

The majority of studies to-date have focused on the relationship between classical SES indicators (such as income, education and occupation), PA and sedentary behaviours (Tandon et al. 2012) but other indicators of social vulnerability, such as children whose parents lack a social network, children from non-traditional families (the child does not live with both parents), migrant children or children with unemployed parents, are rarely explored in the literature. Social vulnerabilities can be defined as social (e.g. migrant) and economic (e.g. unemployment) situations that can increase the susceptibility to harm and that eventually amount to social disconnectedness (Haudenhuyse et al. 2012). These social vulnerable groups tend to adopt unhealthier behaviours and to be less active compared to non-vulnerable groups (Hawkins et al. 2009; Labree et al. 2014). We hypothesized that children from vulnerable groups would have lower levels of PA and higher levels of ST compared to non-vulnerable groups due to financial constraints and negative experiences faced by vulnerable children. Some investigations reported that migrant children had lower levels of PA compared to native children as a result of the acculturation and a different body image perception (Labree et al. 2014). Non-traditional families could be at risk of being more inactive and of having lower sports participation levels because they might have lower modelling abilities and financial capacity compared to traditional families (Quarmby et al. 2011). Children with unemployed parents reported lower levels of PA and higher levels of ST compared to children with employed parents

100 (Federico et al. 2009). Job loss raises TV-watching and since parents exert an impact on 101 children, this may negatively affect children. Finally, we expect that children whose 102 parents lack a social network could have a lower participation in PA and higher ST levels 103 because of less access to resources and personal contact that could encourage activity 104 levels. 
To our knowledge no studies have examined a set of social vulnerabilities in the same population. Four vulnerable groups were investigated: children whose parents lack a social network; children from non-traditional families; migrant children and children with either one or both parents unemployed. This paper aims to explore (i) the cross-sectional and prospective associations between being a member (vs. non-member) of a vulnerable group at baseline and PA (reported and objectively-assessed with accelerometers), sports

111 club membership and ST, at two time points, in European children and (ii) the association

112 of accumulated vulnerability (belonging to multiple vulnerable groups) with PA, sports

113 club membership and ST at baseline. This will allow us to understand whether the

114 disadvantages of socio-economic circumstances in European children are associated with 115 unhealthy activity behaviours.

\section{Methods}

119 Design and study population

120 IDEFICS is a multi-centre prospective cohort study, including a school- and community121 based obesity prevention intervention in eight European countries (Belgium, Cyprus, 122 Estonia, Germany, Hungary, Italy, Spain and Sweden). At baseline (T0), 16,228 children 123 aged 2.0-9.9 years were examined from September 2007 to June 2008 (response rate $12451 \%$ ). The first follow-up (T1) took place two years later (September 2009-June 2010) 125 when 11,038 children aged 4.0-11.9 years were re-examined. In all survey centres, 126 recruitment was carried out at the community level. Parents of children eligible for 127 inclusion were identified and recruited through local kindergartens and schools. The 128 survey comprised anthropometrical measurements, examinations of children and parental 129 self-completion questionnaires on lifestyle habits and dietary intakes of children. 130 Standardised procedures were used by all survey centres. A detailed description is given 131 by Ahrens et al. (Ahrens et al. 2011).

132 Parents or legal guardians gave written informed consent for examinations and data 133 collection for their children, while children expressed oral consent. Ethical approval was 134 obtained from the research ethics authority of each participating centre. 
A parental questionnaire was used to collect a proxy measure of children's subjectivelymeasured PA and ST (Burdette et al. 2004). Parents reported the total hours and minutes children spent playing outdoors during weekends and weekdays and the weekly duration their children spent doing sport in a sports club for a typical week in the previous month. Reported PA was calculated as: [(hours playing outdoors on weekdays*5)+(hours playing outdoors on weekend days*2)+weekly sports participation]/7. Thereafter, participants were classified depending on whether they met the current PA guidelines of $<1 \mathrm{~h} / \mathrm{d}$ vs. $\geq 1 \mathrm{~h} / \mathrm{d}$ (World Health Organization 2010). Parents also reported children's sport club membership (dichotomized into belonging or not belonging to a sport club). Moreover, parents reported hours of TV/DVD/video viewing and computer/gamesconsole use for weekdays and weekend days by their children. Response options were: not at all; $<0.5 \mathrm{~h} / \mathrm{d} ;<1 \mathrm{~h} / \mathrm{d} ; 1-<2 \mathrm{~h} / \mathrm{d} ; 2-<3 \mathrm{~h} / \mathrm{d} ;$ and $>=3 \mathrm{~h} / \mathrm{d}$. Total ST per day was calculated as: $\left(5^{*}\right.$ weekday values $+2 *$ weekend values $) / 7$. Participants were divided into two groups depending on whether they met current ST guidelines of $\leq 2 \mathrm{~h} / \mathrm{d}$ vs. $>2 \mathrm{~h} / \mathrm{d}$ (American Academy of Pediatrics 2001).

\section{Objectively-measured MVPA}

155 Children were instructed and asked to wear a uniaxial accelerometer (ActiGraph or ActiTrainer, ActiGraph, Pensacola, FL, USA) on a hip belt for at least two days including one weekend day and one-week day (weekdays were weighted by five and weekend days by two and the sum was divided by seven). An average of 730 minutes of valid time was obtained in the final sample. To obtain comparable data despite differing valid times,

160 adjusted MVPA was calculated by dividing raw minutes of MVPA by wear time and multiplying by 730 (Konstabel et al. 2014). Only children with a minimum duration of

$1628 \mathrm{~h}$ monitoring time per day were considered, where non-wear time was defined as at least

$16320 \mathrm{~min}$ of consecutive zeroes. The sampling epoch was set to $15 \mathrm{~s}$ but data were reintegrated into 60 second epochs for analysis. The duration of MVPA was determined according to the cut-offs of Evenson (Evenson et al. 2008).

\section{Classical SES indicators as possible confounder}

168 Education: parents indicated the highest level of education of both themselves and their

169 partners. The particular response categories for each country were coded according to the

170 International Standard Classification of Education (ISCED 1997) and re-categorized into:

171 low (ISCED level 0-2), medium (ISCED level 3-4) and high (ISCED level 5-6)

172 educational levels (UNESCO. 1997). 
Income: parents provided information on the monthly net income of the household after taxes and deductions responding to nine country-specific categories (1: lowest income category to 9:highest income category). The category cut-offs were country-specific according to a scheme based on the median equivalent income, thus guaranteeing comparability between countries. The result was organised into three categories: low (13), medium (4-6) and high (7-9) income.

179 Occupation: parents indicated their occupational position with 18 possible options, which

180 were later transformed into the three-class version of the European Socioeconomic 181 Classification (ESeC): working class, intermediate and salaried (Harrison and Rose 182 2006).

183 For occupation and education, the highest level of either the mother or the father was considered for the purpose of the study.

Vulnerable groups as predictors

187 Four vulnerabilities (dichotomised as vulnerable vs. non-vulnerable) were defined as our main exposures using baseline information from parent-reported questionnaires:

189 Social network: based on the Single Item Measure of Social Support developed by Blacke 190 and McKay (1986) parents were asked how many persons they could rely on in case of 191 need including their family. A minimal social network (vulnerable group) was assessed if the parental answer on the question was either 'Nobody' or '1 person'. Further answer categories were '2-3 persons' and 'more than 3 persons' and were labelled as nonvulnerable (Bammann et al. 2013). This measure has been strongly associated with a composite social support index (Blake and McKay 1986).

196 Family structure: If the child did not live with both his/her parents, the family was defined

197 as a 'non-traditional family' (including single-parent families, stepparent families, living with grandparents or foster parents or in an institution).

199 Origin of parents: A migrant background (vulnerable group) was assumed if one or both parents were born in a country different from where the study took place.

201 Employment status: If at least one of the parents was unemployed or living on social 202 assistance or welfare, the child was considered as belonging to the vulnerable group.

204 A total vulnerability score was calculated by adding up the numbers of vulnerabilities a 205 child was exposed to. Six vulnerability indicators (minimal social network, non206 traditional family, migrant, unemployed, low-income and low-education) were 207 considered. Occupation status was not included as it was highly correlated with 
employment status. The vulnerability score ranged from 0 (the child had no vulnerabilities) to 6 (the child had all six vulnerability indicators) and was divided into

210 four categories (three to six vulnerabilities, two vulnerabilities, one vulnerability and no 211 vulnerability).

\section{Weight categories}

214 Anthropometric measurements were assessed at T0 according to standardised procedures 215 in all participating countries. Barefoot body height was measured to the nearest $0.1 \mathrm{~cm}$ 216 by trained staff using a portable stadiometer (SECA 225). Body weight in $\mathrm{kg}$ was 217 measured by a child-adapted version of electronic scale TANITA BC 420 SMA with the 218 children weighted in a fasting state and wearing only light clothes. Body mass index 219 (BMI) was calculated by dividing body weight in kilograms by squared body height in 220 metres and then transformed into an age- and gender-specific z-score (Cole et al. 1998). 221 Weight groups were categorised using age and gender-specific cut points according to the 222 criteria of the International Obesity Task Force (Cole and Lobstein 2012).

\section{Sample size}

224 Two analysis datasets were defined, one for the subjective and one for the objective measurements. Regarding the subjective measurements, 13,891 children were included for the cross-sectional analysis and 8482 children for the longitudinal analysis after excluding children with missing values in any of the outcomes (see figure 1). Children lost to follow-up belonged more often to the minimal social network group (12.0\% vs. $9.0 \%)$, to non-traditional families ( $25 \%$ vs. $18.4 \%$,), migrants (16.5\% vs. $12.8 \%)$ and unemployed parents $(7.0 \%$ vs. $4.8 \%)$ than those included in the present study.

231 Concerning objective measurements, 9,021 children had at least some valid accelerometer data at T0 but only 5,892 children met the following quality requirements (Konstabel et

233 al. 2014): having at least 8 h daily wearing time for at least 2 days ( 1 weekend day and 1 234 weekday) using 60 second epoch. After two years of follow-up, only 2,285 children measured at both T0 and T1 met the accelerometer quality criteria and were included in the longitudinal analysis (see Figure 2). Children lost to follow-up belonged more often

237 to non-traditional families $(21.3 \%$ vs. $16.2 \%)$ and had more often a migrant background $238(16.5 \%$ vs. $12.8 \%)$ than those who were finally included in this study. 
241 Logistic mixed-effects models were used to assess the cross-sectional and longitudinal

242 associations between the four exposures (social network, family structure, migrant origin

243 and employment status) and each outcome (meeting recommendations for objectively-

244 and subjectively- measured PA and ST, sports club membership). The reference category

245 used was the healthiest behaviour for each outcome (subjective PA $\geq 1 \mathrm{~h}, \mathrm{ST} \leq 2 \mathrm{~h}$, sports

246 club membership and objective MVPA $\geq 1 \mathrm{~h}$ ), respectively. The cross-sectional models

247 were adjusted for baseline age, gender, BMI z-score and classical SES indicators; the

248 objectively-measured PA (MVPA) model was additionally adjusted for season. The

249 longitudinal analyses were again adjusted for baseline age, gender, BMI z-score and

250 classical SES indicators, but also for region (intervention versus control region) and

251 baseline outcomes. A further analysis was conducted to estimate the accumulation of

252 vulnerability at T0 and PA (subjectively and objectively assessed), sports club

253 membership and ST. All models included a random kindergartens/school and a random

254 country effect to account for the clustered study design.

255 Respondents with missing socioeconomic information may not be a random subset of

256 population-based survey participants and excluding them may bias study results (Kim et

257 al. 2007). Therefore, missing values of socioeconomic data were coded as a separate

258 category.

259 Before model building, correlations among SES indicators were checked resulting in the

260 exclusion of occupation status in models with employment status as main exposure to

261 avoid collinearity problems.

262 The significance level was set at 0.01 to account at least partially for multiple testing. The

263 analyses were performed using the Statistical Package for the Social Sciences (version

264 22.0; SPSS, Inc.).

265

266 Results

267

268 Table 1 summarises the distributions of predictors and background variables for the three 269 parent reported outcomes (reported PA, ST and sports club membership) at T0 and T1

270 (see Table S1). Older children presented a higher percentage of meeting PA 271 recommendations than younger children (88.6\% and $86.1 \%$ respectively), exceed ST 272 recommendations (19.6\% and 36.9\% respectively), and being a member of a sports club 273 (27.1\% and 58.5\% respectively). By sexes, males had a lower percentage of children 274 reporting $\geq 1 \mathrm{~h}$ of PA $(87 \%)$ and sports club membership (43.7\%) than females (88\% and 
$45 \%$ respectively). By countries, Germany had the highest percentage of children being member of a sports club (58.3\%) and Cyprus the lowest (38.5\%).

277 Table 2 shows the distributions of predictors and background variables for the 278 objectively-measured PA (MVPA) at T0 and T1. The percentage of children reporting $279 \geq 1 \mathrm{~h}$ of MVPA was lower than subjectively-measured PA.

280

281 Children from vulnerable groups and with missing values presented a lower percentage 282 of meeting PA recommendations, a higher percentage of exceeding ST recommendations 283 and a lower percentage as members of a sports club than non-vulnerable groups. 284 Regarding T1, results were similar to T0 (see Table 1).

Table 3 and Table S2 present odds ratio (OR), 99\% confidence interval (CI) and p-values for the models assessing the cross-sectional and longitudinal associations between the four vulnerability indicators at T0 and the reported PA, ST and sports club membership at T0 and T1, respectively. Regarding T0, children whose parents had minimal social networks $(\mathrm{OR}=1.30$, [99\%CI 1.10-1.61]) were more likely not to reach PA recommendations. Migrants $(\mathrm{OR}=1.32$, [99\%CI 1.17-1.48]) and children with unemployed parents $(\mathrm{OR}=1.33$, [99\%CI 1.07-1.66]) were less likely to meet ST recommendation. Those children whose parents had minimal social networks (OR:1.30, [99\%CI 1.10-1.61]), non-traditional families ( $\mathrm{OR}=1.15$, [99\%CI 1.01-1.31]), migrants $(\mathrm{OR}=1.49,[99 \% \mathrm{CI} 1.33-1.68])$ and children with unemployed parents $(\mathrm{OR}=1.34$, [99\%CI 1.06-1.70]) were less likely to belong to a sports club. After two-year follow-up, associations remained for non-traditional families and children with unemployed parents who were less likely to belong to a sports club at $\mathrm{T} 1$.

300 Table 4 shows the models assessing cross-sectional and longitudinal associations between 301 the four vulnerability indicators at T0 and MVPA at T0 and T1, respectively. No associations were found between any of the social vulnerabilities and MVPA at T0 or T1.

303 Table S3 and S4 from supplementary material show the association between the 304 accumulation of vulnerabilities and the four outcomes (reported and objectively-assessed 305 PA, ST and sports club membership) at T0. A higher number of vulnerabilities was not 306 associated with a higher risk of non-compliance with PA recommendations (subjectively 307 and objectively measured) but it was associated with a higher risk of non-compliance with 308 ST recommendations, where the OR increased with the number of present vulnerabilities. 
Likewise, a greater number of vulnerabilities was associated with a lower likelihood of

310 being a member of a sports club.

311 To estimate the change produced when including the classical SES indicators (full

312 adjusted models), we added basic adjusted models (adjusted for baseline age, sex and

313 BMI z-score) as supplementary material (see Table S5). ORs were greater when

314 excluding classical SES compared to the full adjusted models. However, overall results

315 remained unaltered.

\section{Discussion}

320 This paper investigated the association between PA (objectively- and subjectively321 assessed), sports club membership, ST and social vulnerabilities over a two-year period 322 in children aged 2.0-9.9 years participating in a large European cohort study. Vulnerable 323 children presented a higher risk of showing excessive ST cross-sectionally and tended to 324 be less active at sports clubs cross-sectionally and longitudinally, compared to non325 vulnerable groups. Regarding PA, our results did not show a strong association with social 326 vulnerability indicators. Only those children whose parents reported to have minimal 327 social networks were found to be at higher risk of non-compliance with subjectivelyassessed PA recommendations.

360 Adjusting for classical SES indicators allowed investigation of whether the associations 361 between social vulnerabilities and ST/PA were independent of classical SES indicators 362 or whether only the classical SES indicators were finally relevant in the model. We 363 observed that associations may be partly explained by classical SES variables but still 364 independent of classical SES indicators. Therefore, belonging to a vulnerable group 365 seems to be an independent factor of excessive ST and lower participation/activity at 366 sports clubs. A greater effect of the vulnerabilities was observed in cross-sectional 367 analyses as opposed to longitudinal analyses. Consequently, current vulnerability (at the 368 time of outcome assessment) seems the most relevant one for children's PA and ST.

370 The findings of our study are in line with previous research (Gorely et al. 2009; McMillan 371 et al. 2015; Singhammer et al. 2015) despite some differences.

372 Regarding family structure, no significant associations were found between children from 373 non-traditional families and PA or ST, which is in agreement with some studies 374 (McMillan et al. 2015; Singhammer et al. 2015). However, other studies have reported 
that children from non-traditional families accumulate more ST and a higher risk of not meeting PA recommendations as a result of differences in role modelling abilities and

377

378

379

380

381

382

383

384

385

386

387

388

389

390

391

392

393

394

395

396

397

398

399

400

401

402

403

404

405

406

407

408

409

financial capacity (Bagley et al. 2006; Quarmby et al. 2011).

Concerning migrant status, we found statistical differences between migrant children and exceeding ST recommendations at baseline. The acculturation in the host society acquiring Western lifestyle characterized by lower levels of PA and higher levels of sedentary behaviours and different body image perceptions maintained from the country of origin could be the reason of differences found between migrant and native children. However, no association was found between migrant children and not meeting PA recommendations. Similar to our study, Puder et al., (2013) showed that migrant children had a significantly higher amount of ST compared with children born in the country of measurement. Contrary to what we observed, it was showed that PA levels in children were significantly lower among migrant children compared to children in the native population (Labree et al. 2014).

Children whose parents were unemployed were more likely to exceed ST recommendations at baseline compared to non-unemployed parents. These conclusions were confirmed by previous papers (Hawkins et al. 2009; van Rossem et al. 2012). Unemployed people are at a higher risk of depression and inactivity compared to employed people. Since parents are important role models for children this could lead to lower activity levels in children (Van Domelen et al. 2011). Nonetheless, our results did not show any association between children with unemployed parents and being at higher risk of not meeting PA recommendations, like other investigations have demonstrated (Federico et al. 2009).

Children whose parents had minimal social networks had a higher risk of non-compliance with PA recommendations (subjectively-assessed) at baseline but they did not show a higher risk of exceeding ST recommendations. Not only parents but their networks can influence children's behaviours. Therefore, children whose parents have large social networks could have a positive influence for performing higher levels of PA. To our knowledge, no studies have investigated the associations between parent's social network and children's PA and ST.

In line with previous studies, we found that all vulnerable groups were less likely to participate at sports clubs than children from non-vulnerable groups at baseline and 
follow-up (McMillan et al. 2016; Toftegaard-Støckel et al. 2010). These associations

411 were rather weak for children from non-traditional families and higher for children with

412 unemployed parents.

414 Some limitations of the present study should be acknowledged. Firstly, the IDEFICS

415 study is not representative of the European population nor of the participating countries.

416 Each survey centre only covered a delimited geographic area within a country making

417 extrapolation of the results difficult and only a sub-sample of the participants wore an

418 accelerometer. Furthermore, a selection bias cannot be precluded as the children lost to

419 follow-up had more social vulnerabilities at baseline and as voluntary participation

420 might be less frequent from very high or very low SES families. Besides, since self-

421 reported PA usually overestimates total PA compared to accelerometers, subjective PA

422 data should be interpreted with caution. It is questionable how reliably the duration of

423 outdoor-play and sports club membership capture total PA and how reliable the

424 dichotomization of meeting the PA guidelines is according to self-reported PA. On the

425 other side, accelerometers may underestimate the overall activity because they cannot

426 accurately capture activities that are not step-based (such as swimming or cycling)

427 (Colley et al. 2011). Therefore, MVPA may be diminished, which may partly explains

428 the current results as associations would be attenuated. Moreover, valid data on

429 accelerometers was considered when children had at least two days of recording time

430 (including one weekend day and one-week day) with a minimum 8-hour duration of

431 monitoring time per day, which could be insufficient for a correct assessment of

432 whether they meet the PA guidelines. Finally, even though we have controlled for

433 several potential confounders, we cannot preclude unmeasured confounding e.g.

434 through parents' health status, parents' mental health and other socio-cultural factors.

435

436 A particular strength of this study is that to our knowledge, no research has been done 437 concerning the association of vulnerabilities such as social network, family structure and 438 unemployment status with objectively- and subjectively-assessed PA and ST in children 439 in a longitudinal study. Having two measures of PA (subjectively and objectively440 assessed) provide different information. For example, sports club participation usually 441 requires regular payments and it has hence other barriers than playing on a playground.

442 Accelerometers could register both activities but it could not distinguish these differences.

443 The large sample size of eight countries following standardised procedures is also a 444 strength. 
446 Future studies may investigate children with a different country of origin and family

447 structure in more depth to help identify children at higher risk of low PA and high ST.

448 Moreover, more studies including both subjective and objective measures of PA levels

449 and sedentary behaviours are needed to test different constructs which provide additional

450 information and compare possible discrepancies in results to analyse the causes.

451

\section{Conclusion}

453

454 The results suggest a higher risk for excessive ST cross-sectionally in children with unemployed parents and migrants as well as lower odds of being a member in a sports club cross-sectionally and longitudinally in all vulnerable groups independent of family income, parental occupation and parental education. However, no associations were found between any of the social vulnerabilities and objectively-assessed PA. Policy makers should focus on decreasing ST sedentary behaviours among vulnerable groups as well as on offering subsidised access to external exercise, fitness, sports clubs and facilities.

462

\section{Compliance with Ethical Standards}

464 The authors declare that there are no conflicts of interest regarding this manuscript.

465

466

\section{References}

467

468

469

470

Ahrens W, Bammann K, Siani A, et al. (2011) The IDEFICS cohort: design, characteristics and participation in the baseline survey. Int J Obes (Lond) 35 Suppl 1:S3-15 doi:10.1038/ijo.2011.30

American Academy of Pediatrics (2001) Children, adolescents, and television. Pediatrics 107: 423-426 doi: 10.1542/peds.107.2.423 Atkin AJ, Gorely T, Clemes SA, et al. (2012) Methods of Measurement in epidemiology: sedentary Behaviour. Int J Epidemiol 41:1460-1471 doi:10.1093/ije/dys118

Bagley S, Salmon J, Crawford D (2006) Family structure and children's television viewing and physical activity Med Sci Sports Exerc 38:910-918 doi:10.1249/01.mss.0000218132.68268.f4

Bammann K, Gwozdz W, Lanfer A, et al. (2013) Socioeconomic factors and childhood overweight in Europe: results from the multi-centre IDEFICS study. Pediatr Obes 8:1-12 doi:10.1111/j.2047-6310.2012.00075.x

Blake RL, Jr., McKay DA (1986) A single-item measure of social supports as a predictor of morbidity. J Fam Pract 22:82-84 

measure of physical activity in preschool-aged children. Arch Pediatr Adolesc Med 158:353-357 doi:10.1001/archpedi.158.4.353

Cole TJ, Freeman JV, Preece MA (1998) British 1990 growth reference centiles for weight, height, body mass index and head circumference fitted by maximum penalized likelihoodStat Med 17:407-429

Cole TJ, Lobstein T (2012) Extended international (IOTF) body mass index cut-offs for thinness, overweight and obesity. Pediatr Obes 7:284-294 doi:10.1111/j.20476310.2012.00064.x

Colley RC, Garriguet D, Janssen I, Craig CL, Clarke J, Tremblay MS (2011) Physical activity of Canadian adults: accelerometer results from the 2007 to 2009. Canadian Health Measures Survey Health Rep 22:7-14

Drenowatz C, Eisenmann JC, Pfeiffer KA, Welk G, Heelan K, Gentile D, Walsh D (2010) Influence of socio-economic status on habitual physical activity and sedentary behavior in 8- to 11-year old children. BMC Public Health 10:214 doi:10.1186/1471-2458-10-214

Ekelund U, Luan J, Sherar LB, Esliger DW, Griew P, Cooper A (2012) Moderate to vigorous physical activity and sedentary time and cardiometabolic risk factors in children and adolescents Jama 307:704-712 doi:10.1001/jama.2012.156

Evenson KR, Catellier DJ, Gill K, Ondrak KS, McMurray RG (2008) Calibration of two objective measures of physical activity for children. J Sports Sci 26:1557-1565 doi:10.1080/02640410802334196

Federico B, Falese L, Capelli G (2009) Socio-economic inequalities in physical activity practice among Italian children and adolescents: a cross-sectional study Z. Gesundh Wiss 17:377-384 doi:10.1007/s10389-009-0267-4

Foley LS, Maddison R, Jiang Y, Olds T, Ridley K (2011) It's not just the television: survey analysis of sedentary behaviour in New Zealand young people. Int J Behav Nutr Phys Act 8:132 doi:10.1186/1479-5868-8-132

Hagströmer M, Ainsworth BE, Oja P, Sjöoström M (2010) Comparison of a subjective and an objective measure of physical activity in a population sample. J Phys Act Health 7:541550

Hanson MD, Chen E (2007) Socioeconomic status and health behaviors in adolescence: a review of the literature. J Behav Med 30:263-285 doi:10.1007/s10865-007-9098-3

Harrison E, Rose D (2006) The european socio-economic classification (ESeC) user guide. University of Essex, Colchester, https://www.iser.essex.ac.uk/files/esec/guide/docs/UserGuide.pdf. Accessed 20 July 2016

Hawkins SS, Cole TJ, Law C (2009) Examining the relationship between maternal employment and health behaviours in 5-year-old British children. J Epidemiol Community Health 63:999-1004 doi:10.1136/jech.2008.084590

Haudenhuyse RP, Theeboom M, Coalter F (2012) The potential of sports-based social interventions for vulnerable youth: implications for sport coaches and youth workers. Journal of Youth Studies, 15:4, 437-454, DOI: 10.1080/13676261.2012.663895

Janssen I, Leblanc AG (2010) Systematic review of the health benefits of physical activity and fitness in school-aged children and youth Int J Behav Nutr Phys Act 7:40 doi:10.1186/1479-5868-7-40 
Kim S, Egerter S, Cubbin C, Takahashi ER, Braveman P (2007) Potential implications of missing income data in population-based surveys: an example from a postpartum survey in California. Public Health Rep 122:753-763

Klitsie T, Corder K, Visscher TL, Atkin AJ, Jones AP, van Sluijs EM (2013) Children's sedentary behaviour: descriptive epidemiology and associations with objectively-measured sedentary time. BMC Public Health 13:1092 doi:10.1186/1471-2458-13-1092

532

533

534

535

536

537

538

539

540

541

542

543

544

545

546

547

548

549

550

551

552

553

554

555

556

557

558

559

560

561

562

563

564

565

566

567

568

Konstabel K, Veidebaum T, Verbestel V, et al. (2014) Objectively measured physical activity in European children: the IDEFICS study-.Int J Obes (Lond) 38 Suppl 2:S135-143 doi:10.1038/ijo.2014.144

Labree W et al. (2014) Physical activity differences between children from migrant and native origin. BMC Public Health 14:819 doi:10.1186/1471-2458-14-819

McMillan R, McIsaac M, Janssen I (2015) Family structure as a predictor of screen time among youth. PeerJ 3:e1048 doi:10.7717/peerj.1048

McMillan R, McIsaac M, Janssen I (2016) Family Structure as a Correlate of Organized Sport Participation among Youth. PLoS One 11:e147403 doi:10.1371/journal.pone.0147403

Pate RR, Mitchell JA, Byun W, Dowda M (2011) Sedentary behaviour in youth. Br J Sports Med 45:906-913 doi:10.1136/bjsports-2011-090192

Puder J, Pinto AM, Bonvin A, Bodenman P, Munsch S, Kriemler S, Marques-Vidal P (2013) Health-related quality of life in migrant preschool children BMC Public Health 13:384 doi:10.1186/1471-2458-13-384

Quarmby T, Dagkas S, Bridge M (2011) Associations between children's physical activities, sedentary behaviours and family structure: a sequential mixed methods approach. Health Educ Res 26:63-76 doi:10.1093/her/cyq071

Raudsepp L, Viira R (2008) Changes in physical activity in adolescent girls: a latent growth modelling approach. Acta Paediatr 97:647-652 doi:10.1111/j.1651-2227.2008.00748.x

Singhammer J, Ried-Larsen M, Møller NC, Lund-Kristensen P, Froberg K, Andersen LB (2015) Single parent status and children's objectively measured level of physical activity. Sports Med Open 1:10 doi:10.1186/s40798-015-0020-1

Strong WB, Malina RM, Blimkie et al. (2005) Evidence based physical activity for schoolage youth. J Pediatr 146:732-737 doi:10.1016/j.jpeds.2005.01.055

Tandon PS, Zhou C, Sallis JF, Cain KL, Frank LD, Saelens BE (2012) Home environment relationships with children's physical activity, sedentary time, and screen time by socioeconomic status. Int J Behav Nutr Phys Act 9:88 doi:10.1186/1479-5868-9-88

Toftegaard-Støckel J, Strandbu A, Solenes O, Jørgensen P, Fransson K (2010) Sport for children and youth in the Scandinavian countries. Sport in Society 13: 625-642 doi.org/10.1080/17430431003616332,

UNESCO Institute for Statistics (1997) International Standard Classification of Education (ISCED). UNESCO: Montreal, QC, 2006.

http://www.unesco.org/education/information/nfsunesco/doc/isced_1997.htm. Accessed 15 July 2016.

Van Domelen DR, Koster A, Caserotti P, et al. (2011) Employment and physical activity in the U.S. Am J Prev Med 41:136-145 doi:10.1016/j.amepre.2011.03.019 
van Rossem L, Vogel I, Moll HA, Jaddoe VW, Hofman A, Mackenbach JP, Raat H (2012) An observational study on socio-economic and ethnic differences in indicators of sedentary behavior and physical activity in preschool children. Prev Med 54:55-60 doi:10.1016/j.ypmed.2011.10.016

573 Wang X, Hui Z, Terry PD, et al. (2016) Correlates of Insufficient Physical Activity among Junior High School Students: A Cross-Sectional Study in Xi'an, China. Int J Environ Res Public Health 13 doi:10.3390/ijerph13040397

World Health Organization (2010) Global recommendations on Physical activity for Health. Geneva: World Health Organization. 